\title{
Mechanistic insights into the manganese-dependent phosphodiesterase activity of yeast Dbr1 with bis-p- nitrophenylphosphate and branched RNA substrates
}

\author{
BEATE SCHWER, ${ }^{1}$ FAHAD KHALID, ${ }^{1}$ and STEWART SHUMAN ${ }^{2}$ \\ ${ }^{1}$ Department of Microbiology and Immunology, Weill Cornell Medical College, New York, New York 10065, USA \\ ${ }^{2}$ Molecular Biology Program, Sloan-Kettering Institute, New York, New York 10065, USA
}

\begin{abstract}
Saccharomyces cerevisiae Dbr1 is a manganese-dependent RNA debranching enzyme that cleaves the $2^{\prime}-5^{\prime}$ phosphodiester bond of the lariat introns formed during pre-mRNA splicing. Dbr1 is a member of the binuclear metallophosphoesterase enzyme superfamily. We showed previously via alanine scanning that RNA debranching in vivo and in vitro depends on conserved active site residues His13, Asp40, Asn85, His86, His179, His231, and His233. Here, by extending the alanine scan, we added Cys11 to the ensemble of essential active site components. We report that Dbr1 has a vigorous manganese-dependent phosphodiesterase activity with the non-RNA substrate bis-p-nitrophenylphosphate. Whereas RNA debranching requires His86, bis-p-nitrophenylphosphatase activity does not. We interpret these and other structure-activity relations reported here in light of the crystal structures of Entamoeba Dbr1 and other homologous binuclear metallophosphodiesterases. Our results suggest that (i) Dbr1 adheres to the two-metal mechanism of the enzyme superfamily, but is distinguished by its reliance on a Cys11Xaa-His13 motif to engage one of the catalytic metals instead of the Asp-Xaa-His element typical of other clades within the superfamily; (ii) His86 is a general acid catalyst that protonates the $02^{\prime}$ leaving group of the RNA $2^{\prime}-5^{\prime}$ phosphodiester; and (iii) the favorable $\mathrm{p} K_{\mathrm{a}}$ of $p$-nitrophenol elides the strict need for a general acid during hydrolysis of bis-p-nitrophenylphosphate. The Dbr1 bis-p-nitrophenylphosphatase activity is well suited for high-throughput screening for inhibitors of debranching.
\end{abstract}

Keywords: binuclear metallophosphoesterase; lariat debranching

\section{INTRODUCTION}

Branched RNAs, wherein the $2^{\prime}$ hydroxyl of an internal adenosine nucleotide is linked to the $5^{\prime}$-phosphate end of the intron, are natural end-products of eukaryal pre-mRNA splicing (Padgett et al. 1984; Ruskin et al. 1984). The 2',5'phosphodiester linkages are cleaved by a debranching enzyme Dbr1 (Ruskin and Green 1985; Arenas and Hurwitz 1987; Nam et al. 1994) to yield a linear intron, which is then degraded or processed by nucleases. The DBR1 gene is not essential for viability in Saccharomyces cerevisiae and $d b r 1 \Delta$ cells show no discernible growth defect, despite the intracellular accumulation of lariat intron RNAs (Chapman and Boeke 1991). Schizosaccharomyces pombe $\mathrm{dbr}^{+}$is also nonessential, although the $d b r 1 \Delta$ mutant is slow growing (Nam et al. 1997). In contrast, genetic ablation of mammalian Dbr1 is embryonic lethal (Zheng et al. 2015). Budding and fission yeast $d b r 1 \Delta$ strains have been exploited for RNA-seq experiments that comprehensively identify splicing events ge-

Corresponding authors: bschwer@med.cornell.edu, s-shuman@ski. mskcc.org

Article published online ahead of print. Article and publication date are at http://www.rnajournal.org/cgi/doi/10.1261/rna.058552.116. nome wide (Bitton et al. 2014; Stepankiw et al. 2015; Qin et al. 2016).

Dbr1 is a member of the binuclear metallophosphoesterase enzyme superfamily, the signature features of which are a requirement for transition metal ions for catalysis of phosphodiester or phosphomonoester hydrolysis and a conserved active site wherein histidine, asparagine, and aspartate side chains are the principal metal ligands (Matange et al. 2015). A binuclear metallophosphodiesterase active site is exemplified in the crystal structure of Mycobacterium tuberculosis Rv0805, a manganese-dependent $2^{\prime}, 3^{\prime}$-cyclic nucleotide phosphodiesterase (Keppetipola and Shuman 2008), which includes two divalent metals (one iron and one manganese) and a phosphate anion (Fig. 1A; Shenoy et al. 2007; Podobnik et al. 2009). In the MtuRv0805 structure, a metal-bridging water-the putative nucleophile-is situated $3 \AA$ from the phosphorus atom, in an almost perfectly apical orientation

(C) 2016 Schwer et al. This article is distributed exclusively by the RNA Society for the first 12 months after the full-issue publication date (see http://rnajournal.cshlp.org/site/misc/terms.xhtml). After 12 months, it is available under a Creative Commons License (Attribution-NonCommercial 4.0 International), as described at http://creativecommons.org/licenses/ by-nc/4.0/. 


\section{A MtuRv0805}
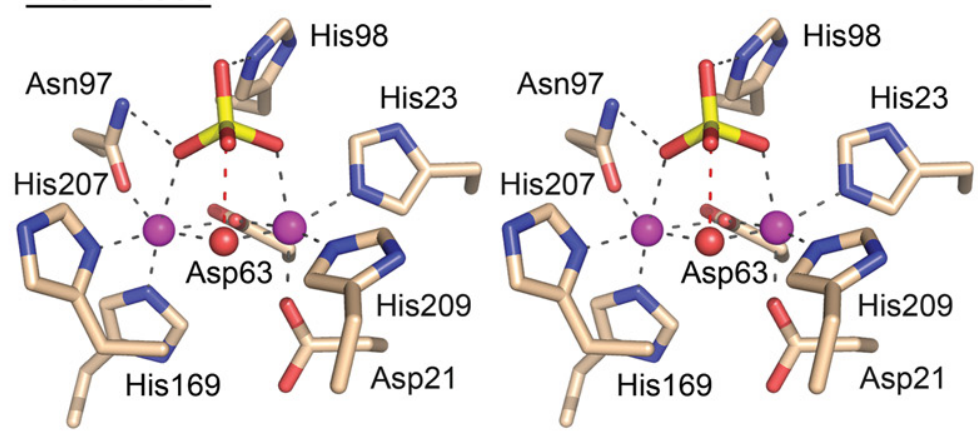

B Dbr1
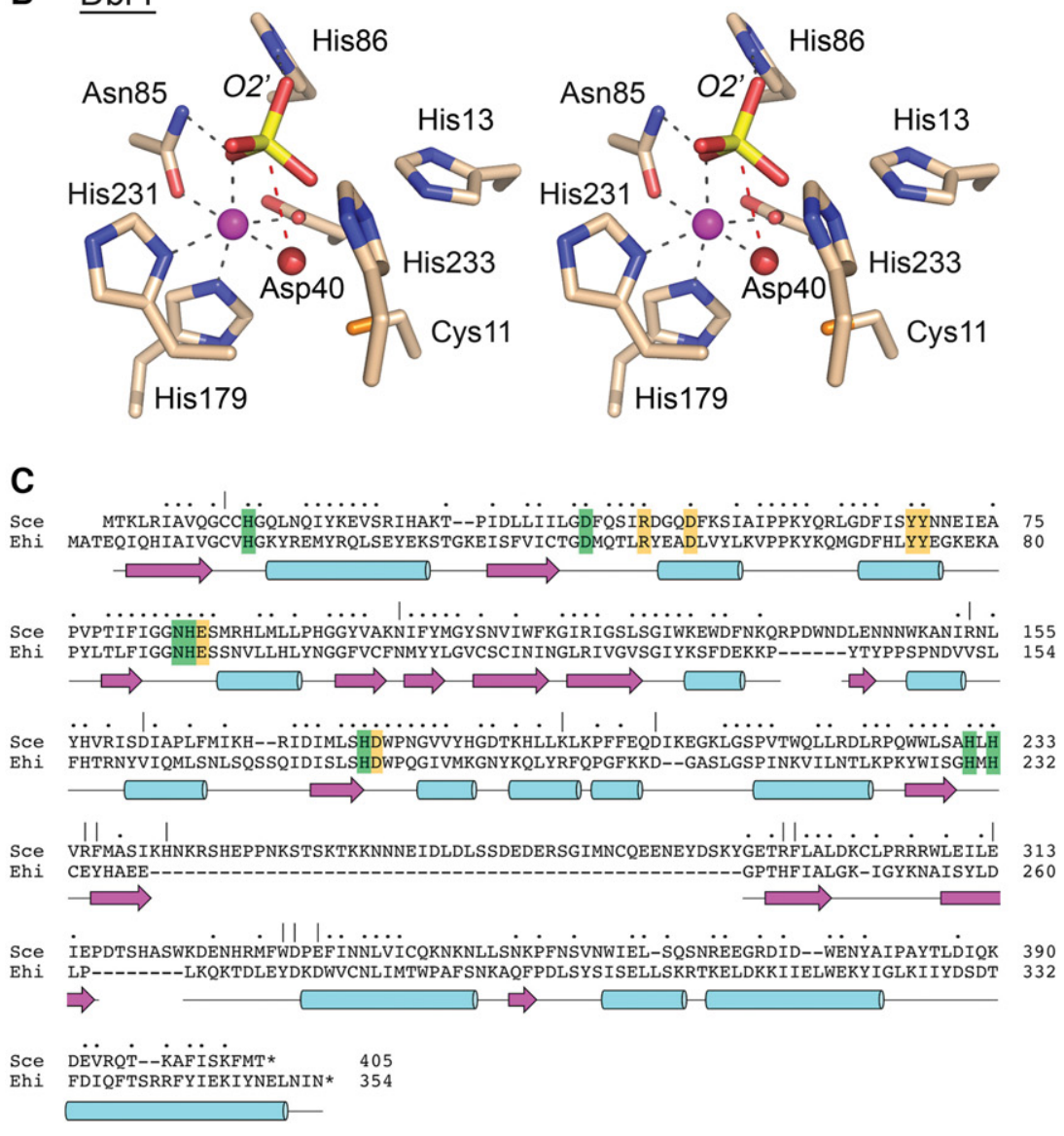

FIGURE 1. Dbrl is a metallophosphoesterase. $(A, B)$ Stereo views of the aligned active sites of $M$. tuberculosis Rv0805 ( $A$; from pdb 2HY1) and E. histolytica Dbr1 ( $B$; from pdb $4 \mathrm{PEH).} \mathrm{The} \mathrm{amino}$ acids (stick models) coordinating the metal ions (magenta spheres) and either a phosphate anion (in Rv0805) or a scissile $2^{\prime}$-phosphate (in Dbr1) are shown. The putative metal-bound water nucleophile is depicted as a red sphere. Amino acids in the EhiDbrl structure are renumbered according to their position in the SceDbrl polypeptide. $(C)$ The amino acid sequences of S. cerevisiae (Sce) and E. histolytica (Ehi) Dbrl are aligned. Gaps in the alignment are indicated by $(-)$. Positions of side chain identity/similarity are denoted by dots. Secondary structure elements of EhiDbr 1 are shown, with $\beta$ strands depicted as magenta arrows and helices as cyan cylinders. Conserved SceDbr1 amino acids identified previously by alanine scanning as important for debranching activity in vivo are shaded either in green (for essential active site constituents) or gold (for amino acids that play a structural role). The SceDbrl amino acids that were subjected to alanine scanning in the present study are denoted by ( | ). to the putative "leaving" oxygen atom. The metal ions, both octahedrally coordinated, are imputed to play two catalytic roles: (i) They each engage one phosphate oxygen and thereby stabilize the presumptive phosphorane transition state; and (ii) they jointly coordinate the water nucleophile and thereby lower its $\mathrm{p} K_{\mathrm{a}}$.

We have shown that purified recombinant S. cerevisiae, S. pombe, and mouse Dbrl proteins are manganese-dependent phosphodiesterases that hydrolyze the $2^{\prime}-5^{\prime}$ linkage of native lariat introns and synthetic branched RNAs (Khalid et al. 2005). Mutagenesis of S. cerevisiae Dbr 1 (SceDbr1; 405 aa) targeting 28 residues conserved in fission yeast, mouse, fruit fly, and plant Dbrl homologs identified 13 amino acids at which alanine substitutions resulted in lariat intron accumulation in vivo (Khalid et al. 2005). Seven of the side chains important for SceDbr1 function (His13, Asp40, Asn85, His86, His179, His231, and His233) are counterparts of amino acids that comprise the active site of Mre11, a DNA 3'$5^{\prime}$ metallophosphodiesterase (Hopfner et al. 2001), CthPnkp, an RNA 2',3' metallophosphoesterase (Wang et al. 2013), and MtuRv0805. Because mutating these residues in SceDbr1 abolished or diminished debranching activity in vitro, we proposed that Dbrl is structurally and mechanistically akin to other binuclear metallophosphoesterases (Khalid et al. 2005).

The crystal structure of Entamoeba histolytica Dbr1 (EhiDbr1; 354-aa) affirmed that the enzyme adopts a metallophosphodiesterase fold, embellished by a distinctive internal loop and a unique C-terminal domain (Montemayor et al. 2014). Two co-crystal structures of EhiDbr1-with 5'-GMP (a debranching reaction product) and with a short RNA with an internal 2'-phosphate (a branchpoint mimetic) - provided the first insights into the basis for RNA branch recognition, because the $5^{\prime}$-phosphate of GMP superimposed on the 2'-phosphate of the RNA oligonucleotide (Montemayor et al. 2014). The EhiDbr1 structure revealed that the branchpoint adenine nucleobase is flipped into a buried pocket wherein it is sandwiched in a $\pi-\pi-\pi$ 
stack between Tyr64 (Tyr59 in SceDbr1) and His16 (His13 in SceDbr1). The guanine base of the 2'-branch nucleotide (corresponding to the $5^{\prime}$ nucleotide of the intron) is surface exposed and is engaged by the enzyme via a bifurcated hydrogen bond to Asp205 (Asp204 in SceDbr1) from the guanine N1 and N2 atoms. The EhiDbr 1 active site, occupied by a manganese ion and the scissile $2^{\prime}$-phosphate, is shown in Figure $1 \mathrm{~B}$ in an orientation aligned to the active site of MtuRv0805. For convenience, the EhiDbr1 amino acids in Figure $1 \mathrm{~B}$ are numbered according to their positions in SceDbr1. A primary structure alignment of EhiDbr1 and SceDbr1 (Fig. 1C) highlights 112 positions of side chain identity and 53 positions of side chain similarity. All 13 residues identified by alanine scanning as important for SceDbr 1 activity in vivo are identical in EhiDbr1 (Fig. 1C). The EhiDbr1 structure verified the prediction (Khalid et al. 2005) that seven of these key SceDbr 1 amino acids are constituents of the active site (His13, Asp40, Asn85, His86, His179, His231, His233; shaded green in Fig. 1A). The other six important side chains (Arg45, Asp49, Tyr68, Tyr69, Glu87, Asp180; shaded gold in Fig. 1A) play structural roles.

Interest in the mechanism and specificity of Dbr1 is spurred by the discovery that genetic ablation of SceDbr1 (or mutations D40A and N85A of the active site) suppresses the cytotoxicity of TDP-43 expression in budding yeast (Armakola et al. 2012). TDP-43 is a human RNA-binding protein found in cytoplasmic inclusions in the spinal neurons of individuals afflicted by amyotrophic lateral sclerosis (ALS) (Buratti 2015). siRNA knockdown of Dbrl ameliorated TDP-43 toxicity in neurons. It was proposed that intron lariat RNAs that accumulate in the cytoplasm when Dbrl is nonfunctional could act as molecular decoys to sequester TDP43 (Armakola et al. 2012). These findings highlight Dbr1 inhibitors as candidate therapeutics for ALS. To that end, Damha and colleagues have pursued synthesis of chemically modified branched RNAs as potential Dbr1 inhibitors. They reported that branched RNAs with a 2'-phosphoramidate linkage are effective Dbr1 inhibitors in vitro and are themselves debranched slowly by Dbrl (Tago et al. 2015). Thus, the phosphoramidate branch analogs represent pseudosubstrate inhibitors. Casting a wider net for Dbrl antagonists as leads for drug discovery would optimally entail highthroughput screening for inhibitors of Dbrl enzymatic activity in vitro. An impediment to screening is the need for chemically synthesized branched RNAs as substrates for Dbrl and the unsuitability of $5^{\prime}$-radiolabeling of RNA and deployment of gel-based assays of debranching activity for high-throughput formats.

It is assumed because Dbr 1 does not hydrolyze nucleic acid $3^{\prime}-5^{\prime}$ phosphodiester linkages that the only substrates that Dbrl can act on are the $2^{\prime}-5^{\prime}$ phosphodiesters found in branched nucleic acids. Yet, characterization of the binuclear metallophosphoesterase CthPnkp, an RNA $2^{\prime}$ and $3^{\prime}$ endprocessing enzyme, taught us that its substrate spectrum embraces non-RNA and non-nucleotide phosphodiesters and phosphomonoesters (Keppetipola and Shuman 2006, 2007). Here we show that SceDbr1 has vigorous phosphodiesterase activity with the non-RNA substrate bis- $p$-nitrophenylphosphate. Dbr1 hydrolysis of bis-p-nitrophenylphosphate requires manganese as the divalent cation cofactor and is ablated by mutating Dbrl active site residues, with the exception of His86. We exploit the Dbr1-H86A mutant to characterize Dbrl's specificity and manganese requirement for binding to branched substrates. New and prior mutational data are discussed in light of the structures of EhiDbrl and related metallophosphodiesterases.

\section{RESULTS}

\section{Bis-p-nitrophenylphosphate is an effective substrate for Dbr1}

Many members of the binuclear phosphoesterase superfamily, including those for which the physiological substrates are known, are capable of hydrolyzing "generic" phosphoester substrates such as $p$-nitrophenylphosphate (a phosphomonoester) or bis-p-nitrophenylphosphate (a phosphodiester). Hydrolysis of these simple inexpensive compounds liberates a chromogenic product $p$-nitrophenol that is easily quantified by its absorbance at $410 \mathrm{~nm}$. Our aim here was to gauge whether S. cerevisiae Dbrl can utilize a non-RNA phosphoester as a substrate. As shown in Figure 2A, we found that reaction of Dbr1 with $15 \mathrm{mM}$ bis-p-nitrophenylphosphate for $15 \mathrm{~min}$ at $22^{\circ} \mathrm{C}$ in the presence of $5 \mathrm{mM}$ manganese resulted in the release of $p$-nitrophenol in amounts proportional to the input enzyme. From the slope of the titration curve from 0 to $2 \mu \mathrm{g}$ enzyme, we calculated that $3.78 \mathrm{nmol}$ of bis-p-nitrophenylphosphate was hydrolyzed per pmol of Dbr1 during the 15 min reaction, which corresponds to a turnover number of $252 \mathrm{~min}^{-1}$. The amount of product formed by $4 \mu \mathrm{g}$ Dbr $1(250 \mathrm{nmol})$ in $15 \mathrm{~min}$ represents hydrolysis of one-third of the input bis-p-nitrophenylphosphate substrate. The kinetic profile of $p$-nitrophenol release by 1 $\mu \mathrm{g}$ Dbr1 during a 60-min reaction with $15 \mathrm{mM}$ bis-p-nitrophenylphosphate is shown in Figure 2B. The initial rate (calculated via linear regression fitting of the data from 0 to $15 \mathrm{~min}$ ) corresponds to a turnover number of $252 \mathrm{~min}^{-1}$. The bis-p-nitrophenylphosphatase activity was optimal at $\mathrm{pH} 7.5$ in either Tris acetate or Tris- $\mathrm{HCl}$ buffer and was virtually nil at $\mathrm{pH} \leq 6.0$ (Fig. 2C). No $p$-nitrophenol release was detected when Dbr1 was reacted with $15 \mathrm{mM} p$-nitrophenylphosphate or thymidine $5^{\prime}$-monophosphate-p-nitrophenyl ester (not shown).

The bis-p-nitrophenylphosphatase activity of Dbrl was strictly dependent on manganese (Fig. 2D). Other divalent metals added at the same concentration $(5 \mathrm{mM}$ magnesium, copper, zinc, calcium, barium, or nickel) were ineffective. Manganese specificity is in keeping with the predominance of "soft" metal contacts to histidine and asparagine nitrogens (and to a cysteine sulfur). We proceeded to conduct a metal 

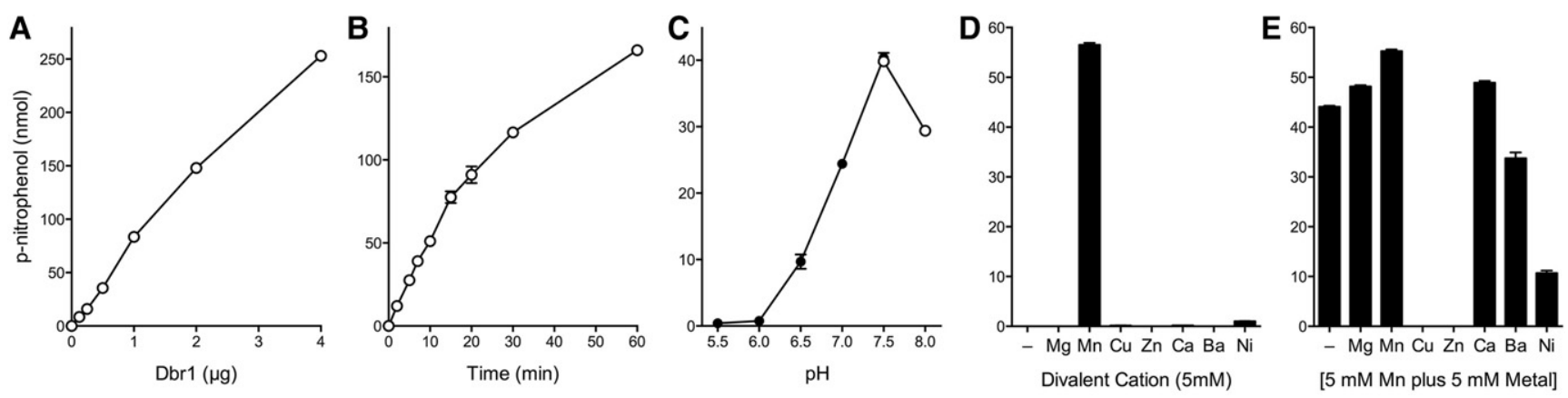

FIGURE 2. Bis- $p$-nitrophenylphosphate is an effective substrate for Dbr1. (A) Dbrl titration. Reaction mixtures ( $50 \mu \mathrm{L})$ containing $50 \mathrm{mM} \mathrm{Tris-HCl}$, $\mathrm{pH} 7.5,5 \mathrm{mM} \mathrm{MnCl}, 15 \mathrm{mM}$ bis-p-nitrophenylphosphate, and 0, 0.125, 0.25, 0.5, 1, 2, or $4 \mu \mathrm{g}$ Dbrl were incubated at $22^{\circ} \mathrm{C}$ for $15 \mathrm{~min}$. The extent of

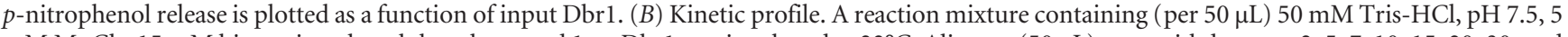
$\mathrm{mM} \mathrm{MnCl} 2,15 \mathrm{mM}$ bis-p-nitrophenylphosphate, and $1 \mu \mathrm{g}$ Dbrl was incubated at $22^{\circ} \mathrm{C}$. Aliquots $(50 \mu \mathrm{L})$ were withdrawn at $2,5,7,10,15,20,30$, and $60 \mathrm{~min}$ and quenched immediately. $p$-Nitrophenol release is plotted as a function of time. $(C) \mathrm{pH}$ dependence. Reaction mixtures $(50 \mu \mathrm{L})$ containing $50 \mathrm{mM}$ Tris buffer (either Tris acetate, $\mathrm{pH}$ 5.0, 5.5, 6.0, 6.5, 7.0, and 7.5; or Tris- $\mathrm{HCl}, \mathrm{pH}$ 7.5, and 8.0), $5 \mathrm{mM} \mathrm{MnCl}$, 12 mM bis-p-nitrophenylphosphate, and $0.75 \mu \mathrm{g}$ Dbrl were incubated at $22^{\circ} \mathrm{C}$ for $20 \mathrm{~min}$. $p$-Nitrophenol release is plotted as a function of $\mathrm{pH}$. $(D)$ Metal requirement and specificity. Reaction mixtures $(50 \mu \mathrm{L})$ containing $50 \mathrm{mM}$ Tris- $\mathrm{HCl}, \mathrm{pH} 7.5,15 \mathrm{mM}$ bis- $p$-nitrophenylphosphate, $1 \mu \mathrm{g}$ Dbr 1 , and either no added metal (-), $5 \mathrm{mM}$ magnesium, manganese calcium or barium (as chloride salts), or $5 \mathrm{mM}$ copper, zinc, or nickel (as sulfate salts) were incubated at $22^{\circ} \mathrm{C}$ for 15

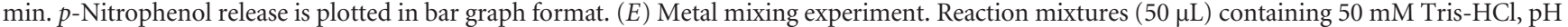
7.5, $5 \mathrm{mM} \mathrm{MnCl}_{2}, 15 \mathrm{mM}$ bis-p-nitrophenylphosphate, $1 \mu \mathrm{g}$ Dbrl, and either no additional metal (-) or $5 \mathrm{mM}$ supplemental metals as specified were incubated at $22^{\circ} \mathrm{C}$ for $15 \mathrm{~min}$. $p$-Nitrophenol release is plotted in bar graph format. (Each datum in the graphs is the average of two independent experiments; error bars denote the range.)

mixing experiment, in which Dbr1 bis- $p$-nitrophenylphosphatase reactions containing $5 \mathrm{mM}$ manganese were supplemented with $5 \mathrm{mM}$ of another divalent cation (Fig. 2E). Copper and zinc abolished Dbrl activity in the presence of manganese, suggesting that these two soft metals might out-compete manganese for one or both metal-binding sites on the enzyme, wherein engaged they are unable to support reaction chemistry. Nickel, also a soft metal, was partially inhibitory in the presence of manganese. In contrast, the "hard" metals magnesium and calcium, which typically engage oxygen atoms on enzymes, had no significant detrimental effect in combination with manganese; nor did barium.

\section{Effects of Dbr1 active site mutations on bis-p-nitrophenylphosphatase activity}

Alanine and conservative mutations were introduced into Dbr1 in lieu of active site residues His13, Asp40, Asn85, His86, His231, and His233. The recombinant mutant Dbr1 proteins were produced in E. coli as $\mathrm{His}_{10}$-tagged fusions and purified from soluble extracts in parallel with wild-type Dbr1. SDS-PAGE affirmed that Dbrl was the predominant polypeptide in the WT and mutant protein preparations (Fig. 3A). (Note that $\mathrm{His}_{10}$ Dbr1 migrates slightly faster, relative to size markers, than its calculated molecular mass of $50 \mathrm{kDa}$.) The proteins were tested for their ability to hydrolyze bis- $p$-nitrophenylphosphate. Whereas alanine mutations H13A, D40A, N85A, H321A, and H323A effaced bis-p-nitrophenylphosphatase activity, the H86A mutant uniquely retained function, generating one-fifth the amount of $p$-nitrophenol product as wild-type Dbr1 (Fig. 3B). Replacing His13, His231, and His233 by Asn or Gln failed to restore bis- p-nitrophenylphosphatase activity. Conservative replacement of Asp40 by Glu or Asn also had no salutary effect. Changing Asn85 to aspartate elicited a slight gain of function versus alanine, to the level of $5 \%$ the activity of wild-type Dbr1; replacing Asn85 with histidine was ineffective. Conservative replacements of His86 by Asn and Gln enhanced bis-p-nitrophenylphosphatase activity vis à vis $\mathrm{H} 86 \mathrm{~A}$, to $42 \%$ and $26 \%$ of wild type, respectively (Fig. 3B). Hydrolysis of bis- $p$-nitrophenylphosphate by the H86N mutant was strictly dependent on exogenous manganese (not shown). These experiments indicate that manganese ligands in the Dbrl active site are critical for hydrolysis of bis-p-nitrophenylphosphate, whereas the His86 side chain that coordinates the scissile phosphodiester is not. This apparent relaxation of requirement for His86 in cleaving the generic substrate bis- $p$-nitrophenylphosphate contrasts with the essentiality of His 86 for the RNA debranching activity of SceDbr1 with a synthetic 31-mer branched RNA substrate (Fig. 4A).

\section{Manganese is required for binding of Dbr1 to a branched RNA substrate}

We exploited the H86A mutant to analyze the RNA-binding properties of $\mathrm{Dbr} 1$ in the presence of its obligate manganese cofactor. Increasing concentrations of Dbr1-H86A (from 4 to $1000 \mathrm{nM}$ ) were incubated for $10 \mathrm{~min}$ at $22^{\circ} \mathrm{C}$ with $5^{\prime}{ }^{32} \mathrm{P}$-labeled synthetic branched 22-mer oligonucleotide (Carriero and Damha 2002; Katolik et al. 2014) composed of a 6-mer $5^{\prime}$ arm and two 8-mers linked to the $\mathrm{O}^{\prime}$ and $\mathrm{O}^{\prime}$ ribose atoms of the branchpoint adenosine (Fig. 4B). RNA binding was assayed via the two-filter method (Wong and Lohman 1993; as adapted by Tanaka and Schwer 2005) and the 

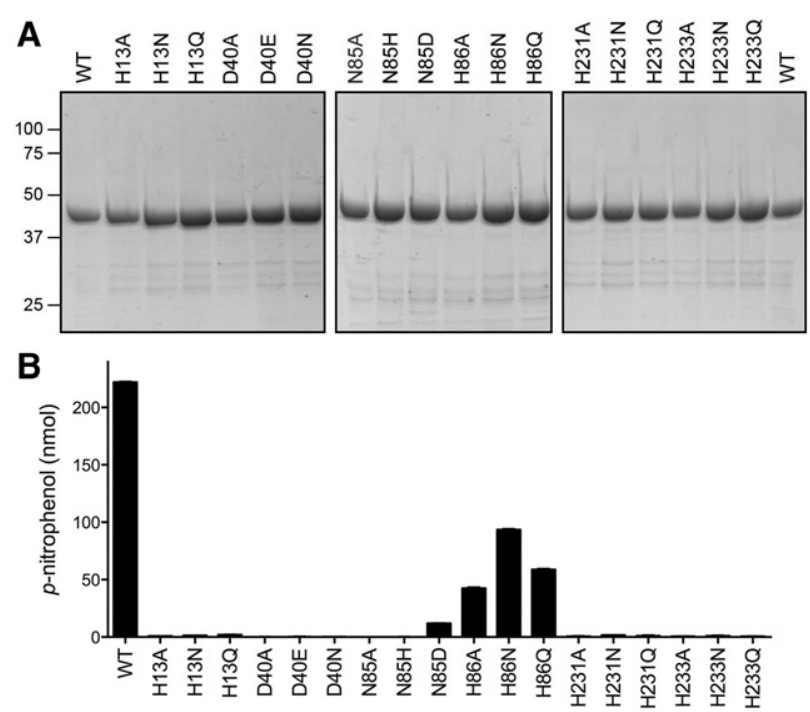

FIGURE 3. Effect of Dbr1 active site mutations on bis-p-nitrophenylphosphatase activity. (A) Dbr1 mutants. Aliquots $(5 \mu \mathrm{g})$ of the heparin Sepharose preparations of wild-type Dbr1 (WT) and the indicated Dbr1 mutants were analyzed by SDS-PAGE. The Coomassie blue-stained gel is shown. The positions and sizes $(\mathrm{kDa})$ of marker proteins are indicated at the left. (B) Reaction mixtures $(50 \mu \mathrm{L})$ containing $50 \mathrm{mM}$ Tris- $\mathrm{HCl}$, $\mathrm{pH} 7.5,5 \mathrm{mM} \mathrm{MnCl}_{2}, 15 \mathrm{mM}$ bis-p-nitrophenylphosphate, and 1.5 $\mu \mathrm{g}$ wild-type or mutant Dbr1 as specified on the $x$-axis were incubated at $22^{\circ} \mathrm{C}$ for $60 \mathrm{~min} . p$-Nitrophenol release is plotted in bar graph format. Each datum is the average of two independent experiments; error bars denote the range.

binding isotherm is shown in Figure $4 \mathrm{~B}$ (branch $+\mathrm{Mn}$ ). The apparent $K_{\mathrm{d}}$ for Dbr1-H86A for the 22-mer branched ligand, calculated by nonlinear regression fitting of the data in Prism, was $53 \mathrm{nM}$. It was reported previously that Dbr1-H86A bound in the presence of manganese to a shorter 7-mer branched RNA (with a trinucleotide $5^{\prime}$ arm and two dinucleotide branches) with a $K_{\mathrm{d}}$ of $507 \mathrm{~nm}$ (Garrey et al. 2014). We surmise that a critical length of one or more of the three component strands is needed for high-affinity binding of Dbr1 to a branched RNA. The salient finding here was that binding of Dbr1-H86A to the 22-mer branch was effaced by omission of manganese from the reaction mixture (Fig. $4 \mathrm{~B}$, branch - Mn), implying that the divalent cation cofactor needs to be engaged in the Dbr1 active site to nucleate formation of a branch-binding pocket. Dbr1-H86A did not bind to a $5^{\prime 32} \mathrm{P}$-labeled linear 12 -mer oligonucleotide in the presence or absence of manganese (Fig. 4B).

Additional binding experiments were performed with a much longer $5^{\prime 32} \mathrm{P}$-labeled synthetic branched 53-mer oligonucleotide composed of a 31-mer $5^{\prime}$ arm and two 11-mers linked to the branchpoint adenosine (Fig. 4C). Whereas the extent of Dbr1-H86A binding at saturation to the 53-mer branch in the presence of manganese was higher than to the 22-mer branch, the 53-mer binding isotherm fit to a similar $K_{\mathrm{d}}$ value of $54 \mathrm{nM}$ (Fig. 4C). A key difference revealed with the longer branch was the capacity of Dbr1-H86A to bind to the longer substrate in the absence of manganese, albeit with apparently lower affinity (approximately eightfold) compared to that in the presence of manganese. In the same vein, we found that Dbr1-H86A could bind to a longer $5^{\prime}$ ${ }^{32} \mathrm{P}$-labeled 41-mer linear RNA in the presence of manganese, though with lower affinity (fourfold) compared to its binding to the 52-mer branch in the presence of manganese. Omission of manganese reduced (by approximately fivefold)
A

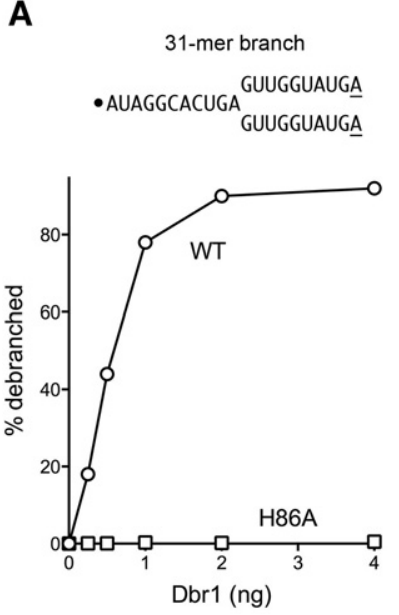

B
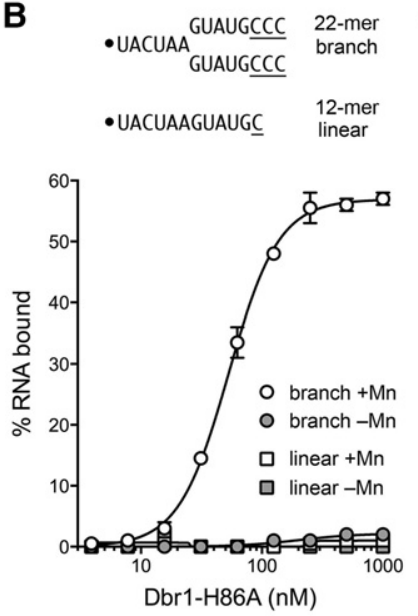
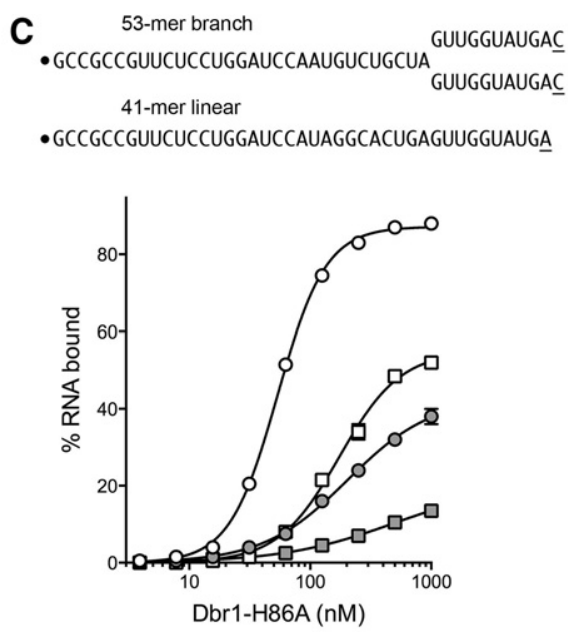

FIGURE 4. RNA-binding properties of Dbr1-H86A. (A) Debranching assay. Reaction mixtures $(20 \mu \mathrm{L})$ containing $50 \mathrm{mM} \mathrm{Tris-HCl,} \mathrm{pH} \mathrm{7.0,} 4 \mathrm{mM}$ $\mathrm{MnCl}_{2}, 2.5 \mathrm{mM}$ DTT, $100 \mathrm{fmol} 5^{\prime 32} \mathrm{P}$-labeled branched 31-mer oligonucleotide (depicted above the graph), and 4 ng wild-type Dbr1 or H86A mutant as specified were incubated at $22^{\circ} \mathrm{C}$ for $10 \mathrm{~min}$. The reactions were quenched by adding $20 \mu \mathrm{L}$ formamide dye mix. The products were analyzed by denaturing PAGE. The ${ }^{32} \mathrm{P}$-labeled RNAs were visualized and quantified by scanning the gel with a phosphorimager. The amount of linear product as a percentage of total RNA is plotted as a function of input Dbr1. $(B, C)$ RNA binding. Increasing concentrations of Dbr1-H86A were incubated with ${ }^{32} \mathrm{P}-$ labeled branched or linear RNAs (depicted above the graphs) in the absence or presence of $5 \mathrm{mM} \mathrm{MnCl}_{2}$ and then applied to a dual nitrocellulose/ nylon membrane filter. The percentage of RNA bound is plotted as a function of protein concentration (logarithmic scale). Each datum is the average of two independent experiments; error bars denote the range. The symbols in panel $C$ are the same as in panel $B$. 
the binding of Dbr1-H86A to the linear 41-mer (Fig. 4C). These experiments suggest that Dbr1 can bind to the extended 5' RNA arm of the longer branched RNA (and the longer linear RNA) in a manner that does not require the branch structure, yet is still aided by the inclusion of manganese (which likely stabilizes the structure of the active site and any adjacent RNA-binding surface).

\section{New round of mutagenesis identifies Cys11 as critical for Dbr1 function}

In modeling the Dbr 1 active site on that of Mre11 (or CthPnkp), we had proposed that Cys11 of Dbr1 occupies the position held by an essential aspartate that coordinates one of the two catalytic manganese ions (Khalid et al. 2005). The crystal structure of EhiDbr1 affirmed that the cysteine is at the same active site position as the canonical aspartate of other metallophosphoesterases (Fig. 1B) and that mutating the EhiDbr1 cysteine (Cys14) to serine eliminated its debranching activity with a synthetic RNA substrate (Montemayor et al. 2014). This position is conserved as a cysteine in Dbr1 homologs from diverse eukaryal taxa. To gauge its functional relevance in yeast Dbr1, we mutated Cys11 to alanine, serine, histidine and aspartate, cloned the mutant DBR1 alleles into TRP1 CEN plasmids under the control of the native $D B R 1$ promoter, and introduced the $D B R 1$ plasmids into dbr1 $\Delta$ cells. To determine whether the mutated Dbr1 proteins were functional, total RNA was extracted from each of the strains and analyzed by Northern blotting with probes specific for the intron sequences of RPS13 and ACT1 pre-mRNAs (Fig. 5B,C). Northern analysis with a probe for the exon sequences of PGK1 mRNA served as a loading control (Fig. 5A), as did ethidium bromide staining of the gel for ribosomal RNAs prior to transfer of the gel contents to a membrane (Fig. 5D). As expected, the steady-state levels of branched intron RPS13 and ACT1 RNAs were greatly increased in $d b r 1 \Delta$ cells compared to wild-type cells. For the purpose of quantification, we set the amount of RPS13 and CYH2 intron RNA detected in dbr1 1 cells to $100 \%$. The amounts of branched intron RNAs in wild-type cells were $4 \%$ of the levels in $d b r 1 \Delta$ cells. Dbr1 mutants C11A, C11S, and $\mathrm{C} 11 \mathrm{H}$ were apparently nonfunctional, insofar as the steady-state ACT1 and RPS13 intron levels in C11A, C11S, and $C 11 H$ cells were similar to those in $d b r 1 \Delta$ cells. We noted that the extent of intron accumulation in C11D cells was slightly less than in $d b r 1 \Delta$ (61\% for RPS13 and $82 \%$ for $A C T 1)$ and in the three other C11 mutant strains, suggesting that the Dbr1-C11D protein might have some residual bio- activity. Assay of recombinant C11A, C11S, C11H, and C11D proteins for bis- $p$-nitrophenylphosphatase activity in parallel with wild-type Dbr1 showed that C11A, C11S, and $\mathrm{C} 11 \mathrm{H}$ were inert, whereas the $\mathrm{C} 11 \mathrm{D}$ protein was $7 \%$ as active as wild-type Dbr1 (Fig. 5E). We conclude that the Dbr1 mechanism has evolved to use cysteine specifically in lieu of the aspartate found in non-Dbr1 members of the metallophosphoesterase superfamily.

We also expanded the alanine scan of SceDbr1 to target 14 new residues: Asn104, Arg153, Asp162, Lys196, Asp204, Arg235, Phe236, His242, Arg295, Phe296, Glu313, Trp332, Asp333, and Glu335 (denoted by | in Fig. 1C). These amino acids were chosen based on conservation in fission yeast, plant, and/or metazoan Dbr1 homologs using a primary structure alignment generated prior to the publication of the EhiDbr 1 tertiary structure. Nine of the 14 targeted amino acids are conserved in EhiDbr1 (Fig. 1C). Testing of the DBR1-Ala alleles for bioactivity in the $d b r 1 \Delta$ strain by Northern analysis of intron levels showed that $N 104 A$, R153A, D162A, K196A, D204A, R235A, H242A, R295A, E313A, W332A, D333A, and E335A cells showed no significant debranching defect, by the criterion that $A C T 1$ and RPS13 intron levels were $\leq 10 \%$ of the $d b r 1 \Delta$ strain (Fig. $5 \mathrm{~B}, \mathrm{C})$. We surmise that these 12 side chains are not essential for Dbr1 function in vivo. The results are noteworthy in that the inessential residues include Asp204, the counterpart of EhiDbr1 Asp205 that engages the guanine base of the pG nucleotide at the $2^{\prime}-5^{\prime}$ branch (Montemayor et al. 2014). 
In contrast, the F296A mutant was functionally compromised, insofar as the ACT1 and RPS13 intron levels in F296A cells were $61 \%-63 \%$ of the levels observed in $d b r 1 \Delta$ cells. Debranching was less adversely affected in F236A cells, where intron levels were $16 \%-21 \%$ of those in the $d b r 1 \Delta$ strain. The Phe296 equivalent in EhiDbr1 (Phe244) is buried in the hydrophobic core of the protein, leading us to infer that the F296A mutation exerts a detrimental structural effect. The counterpart of Phe236 in EhiDbr1 (Tyr235) is adjacent to Phe244.

\section{DISCUSSION}

Here we extended our genetic and biochemical characterization of yeast Dbr1, the enzyme responsible for debranching the lariat intron products of pre-mRNA splicing. Our results impact on two aspects of scientific interest in Dbrl: (i) screening for Dbr1 inhibitors, which have been suggested as candidates for treatment of ALS; and (ii) clarification of Dbr1's catalytic mechanism in light of issues raised by the EhiDbr1 crystal structures.

Our demonstration that Dbr1 uses bis- $p$-nitrophenylphosphate as a phosphodiesterase substrate enables a simple colorimetric assay for high-throughput screening of chemical and natural product libraries based on inhibition of $p$-nitrophenol release. The strict reliance of Dbrl's bis- $p$-nitrophenylphosphatase activity on manganese, and the ablation of bis- $p$-nitrophenylphosphatase activity by mutations of the Dbr 1 active site, mirror the requirements for, and mutational effects on, Dbrl's RNA debranching activity in vivo and in vitro (Khalid et al. 2005). This concordance suggests that inhibitors of Dbr1's bis-p-nitrophenylphosphatase activity will likely inhibit its debranching activity when subjected to secondary screening in vitro using synthetic branched RNA substrates.

A key difference between the RNA debranching and bis- $p$ nitrophenylphosphatase activities of Dbr1 pertains to the requirement for His86 for the former, but not the latter function. Yeast DBR1-H86A, H86N, and H86Q strains phenocopy $d b r 1 \Delta$ with respect to intron accumulation (Khalid et al. 2005) and the Dbr1-H86A enzyme is grossly defective for catalysis of RNA debranching in vitro, notwithstanding that it binds to synthetic branched RNAs with high affinity. The requirement for His86 is relaxed when bis-p-nitrophenylphosphate is the substrate, insofar as the H86A, H86N, and H86Q proteins retain $20 \%-40 \%$ bis- $p$-nitrophenylphosphatase activity compared to wild-type Dbr1. The structure of EhiDbr1 in complex with a short RNA with an internal $2^{\prime}$ phosphate revealed that His91-Ne (i.e., His86-Ne in yeast Dbr1) coordinates the 2'-bridging oxygen of the scissile phosphate (Fig. 1B) and can thereby serve as a general acid catalyst of the phosphodiesterase reaction by donating a proton to the $\mathrm{O}^{\prime}$ leaving atom of the branchpoint adenosine. The residual phosphodiesterase activity of the Dbr1 His86 mutants with bis- $p$-nitrophenylphosphate can be rational- ized by the fact that the $\mathrm{p} K_{\mathrm{a}}$ of $p$-nitrophenol (7.2) is 5-6 $\mathrm{pH}$ units lower than that of the $2^{\prime}-\mathrm{OH}$ leaving group of a branched intron RNA, based on the experimentally determined $\mathrm{p} K_{\mathrm{a}}$ values of 13.4 for the $2^{\prime}-\mathrm{OH}$ group of $3^{\prime}$-AMP (Velikyan et al. 2001) and 12.3 for the $2^{\prime}-\mathrm{OH}$ of an ApG dinucleotide (Acharya et al. 2003). Thus, expulsion of $p$-nitrophenol will be less reliant on an enzymic general acid. The equivalents of His86 make analogous contacts to the scissile phosphate in many other binuclear metallophosphodiesterases, including His98 in MtuRv0805, as shown in Figure 1A.

The striking feature of the series of EhiDbr1 structures (as apoenzyme with a sulfate anion in the active site; as complexed with 5'-GMP, and as bound to a short oligoribonucleotide with an internal $2^{\prime}$-phosphate) was that each active site contained only one manganese ion, octahedrally coordinated to the yeast Dbr1 equivalents of Asp40-O $\delta$, Asn85-N $\delta$,

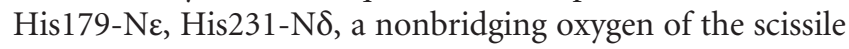
phosphodiester, and the water nucleophile (Fig. 1B). What is clearly "missing" is the signature second metal of the binuclear metallophosphoesterase enzyme family (Matange et al. 2015), which is expected, based on the MtuRv0805 structure (Fig. 1A) and others, to be coordinated octahedrally by SceDbr1 residues Cys11, His13, Asp40, His233, the other nonbridging oxygen of the scissile phosphodiester, and the water nucleophile. One view is that the reported EhiDbr1 structure reflects an incomplete active site. Alternatively, as speculated by Montemayor et al. (2014) and Tago et al. (2015), Dbr1 might diverge mechanistically from other structurally homologous metallophosphoesterase enzymes to catalyze phosphodiester hydrolysis via a single metal cofactor. In the hypothetical single-metal mechanism, the Dbr1 active site Cys is a cysteine thiolate anion that coordinates the water nucleophile together with the single manganese. The cysteine thiolate would thereby act as a general base to abstract a proton from the water (Tago et al. 2015).

We view our findings that mutations of yeast Dbr1 residues Cys11, His13, and His233 (the putative ligands for the second manganese) compromise intron debranching in vivo and efface the hydrolysis of bis- $p$-nitrophenylphosphate as consistent with a canonical binuclear mechanism for Dbr1, with the exception that Dbr1 uses cysteine as one of the ligands for the second metal in lieu of the aspartate found in nonDbr1 binuclear metallophosphoesterases (Matange et al. 2015). We speculate that there are plausible explanations for the absence of a second metal in the reported EhiDbr1 structures. First, the EhiDbr1 crystals were grown by mixture with and equilibration to a precipitant solution containing bis-tris buffer at a $\mathrm{pH}$ of 5.5. We showed here and previously (Khalid et al. 2005) that Dbr1 is inactive at pH 5.5 with respect to hydrolysis of either synthetic branched RNA or bis- $p$-nitrophenylphosphate. We speculate that at $\mathrm{pH} 5.5$, His13 and/or His233 might be protonated and thereby unable to coordinate a missing second manganese. Second, the EhiDbr1 protein solution used in the crystallization experiments (concentrated to $0.4-0.7 \mathrm{mM}$ ) was adjusted to 
$1 \mathrm{mM}$ manganese prior to mixture with equal volumes of ligand solution and precipitant solution, such that the net concentration of manganese during initial crystal growth was submillimolar and only in 1.4- to 2.5-fold molar excess over the enzyme. It is possible that the metal-binding sites in EhiDbr1 have distinct affinities for manganese (either inherently, or particularly at acidic $\mathrm{pH}$ ), such that only one of the two sites is filled under the conditions used for crystallization. Third, it is conceivable that Dbr1 fills one of its metal sites with manganese but uses a different metal for the second site, a strategy shared by several other binuclear metallophosphoesterases (Matange et al. 2015), including MtuRv0805, which fills one metal site with manganese and the other with iron (Shenoy et al. 2007). Ultimate clarification of the Dbr1 mechanism will hinge on capturing additional crystal structures of the enzyme under conditions reflective of active catalysis.

\section{MATERIALS AND METHODS}

\section{Dbr1 expression plasmids}

Yeast expression plasmids p358-Dbr1 (CEN TRP DBR1) and bacterial expression plasmids pET16b-DBR1 encoding wild-type Dbr1 or missense mutants of Dbr1 were constructed as described previously (Khalid et al. 2005). The plasmid inserts were sequenced to confirm the presence of the desired mutations and to exclude the acquisition of unwanted coding changes during amplification or cloning.

\section{RNA isolation and Northern blot analysis}

The p358-Dbr1 plasmids were introduced into a W303-derived dbr1s strain (Mata ura3-52 trp1-63 his3-4200 leu2-1 ade2-101 lys2-801 dbr1::LEUE) (Khalid et al. 2005). The empty vector pSE358 (TRP1 CEN) served as a negative control. Trp ${ }^{+}$cells were selected and grown to mid-logarithmic phase $\left(A_{600}=0.6-0.8\right)$. Aliquots (equivalent to $20 A_{600}$ units) were harvested and RNA was isolated using the Qiagen RNA isolation kit according to the vendor's instructions. RNA concentration was calculated on the basis of $A_{260}$. Equivalent amounts $(15 \mu \mathrm{g})$ of total RNA from each sample were resolved by electrophoresis through a $1.4 \%$ agarose/ formaldehyde gel and transferred to a Hybond membrane (Amersham). DNA fragments to be used as probes to detect the $A C T 1$ and RPS13 introns and the PGK1 mRNA were generated by PCR and radiolabeled probes were prepared using a random priming kit (Roche). Hybridization was performed as described previously (Herrick et al. 1990) and the hybridization signal was quantified by scanning the membrane with a phosphorimager (Molecular Dynamics) and visualized by autoradiography.

\section{Expression and purification of recombinant Dbr1 proteins}

The pET16-based plasmids for expression of wild-type His ${ }_{10}$ Dbr1 and the His-tagged Dbr1 mutants were transformed into Escherichia coli strain BL21-Codon Plus(DE3) RIL. Cultures were inoculated from single colonies and maintained in logarithmic growth at $37^{\circ} \mathrm{C}$ in Luria-Bertani medium containing $0.1 \mathrm{mg} / \mathrm{mL}$ ampicillin to a final volume of $500 \mathrm{~mL}$. When $A_{600}$ reached $0.6-0.8$, the cultures were chilled on ice for $30 \mathrm{~min}$, and then adjusted to $0.4 \mathrm{mM}$ isopropyl- $\beta$-D-thiogalactopyranoside (IPTG) and $2 \%$ ethanol. The cultures were incubated for $16-18 \mathrm{~h}$ at $17^{\circ} \mathrm{C}$ with constant shaking. Cells were harvested by centrifugation and the pellets stored at $-80^{\circ} \mathrm{C}$. His ${ }_{10}$ Dbrl proteins were purified from soluble bacterial extracts by sequential Ni-NTA agarose and heparin Sepharose chromatography steps as described previously (Khalid et al. 2005). Protein concentrations were determined using the Bio-Rad dyebinding reagent with BSA as the standard.

\section{Bis-p-nitrophenylphosphatase assay}

Reaction mixtures $(50 \mu \mathrm{L})$ containing $50 \mathrm{mM}$ Tris- $\mathrm{HCl}, \mathrm{pH} 7.5$, $5 \mathrm{mM} \mathrm{MnCl} 2,12$ or $15 \mathrm{mM}$ bis- $p$-nitrophenylphosphate, and Dbr1 as specified were incubated at $22^{\circ} \mathrm{C}$. The reactions were quenched by adding $5 \mu \mathrm{L} 0.5 \mathrm{M}$ EDTA followed by $0.95 \mathrm{~mL}$ of 1 $\mathrm{M} \mathrm{NaOH}$. Release of $p$-nitrophenol was determined by measuring $A_{410}$ and interpolating the value to a $p$-nitrophenol standard curve.

\section{RNA-binding assay}

The double-filter RNA-binding assay (Wong and Lohman 1993) was performed using nitrocellulose (from Bio-Rad) and Hybond-N+ nylon (Amersham) membranes pretreated as described previously (Tanaka and Schwer 2005). A 96-well dot-plot apparatus (Schleicher and Schuell) was assembled so that the nylon membrane was placed underneath the nitrocellulose filter. Binding reaction mixtures (50 $\mu \mathrm{L})$ containing $40 \mathrm{mM}$ Tris- $\mathrm{HCl}, \mathrm{pH} 7.5,2 \mathrm{mM}$ DTT, $40 \mathrm{pM}$ $(\sim 10,000 \mathrm{cpm}){ }^{32} \mathrm{P}$-labeled nucleic acid, either $5 \mathrm{mM} \mathrm{MnCl}_{2}$ or no added metal, and varying amounts $(0-1 \mu \mathrm{M})$ of Dbr1-H86A were incubated for $10 \mathrm{~min}$ at $22^{\circ} \mathrm{C}$. The mixtures were applied to the dotblot sample wells under vacuum and the filter wells were washed twice with $100 \mu \mathrm{L}$ aliquots of ice-cold buffer containing $40 \mathrm{mM}$ Tris- $\mathrm{HCl}, \mathrm{pH}$ 8.0, 1 mM EDTA, 10\% glycerol. The radiolabel adsorbed to the nitrocellulose membrane (Dbr1-bound RNA) and nylon membrane (free RNA) was quantified using a Phosphorimager and ImageQuant software (Molecular Dynamics).

\section{ACKNOWLEDGMENTS}

This work was supported by National Institutes of Health grants GM102961 (B.S.) and GM46330 (S.S.). We are grateful to Drs. Sandra Carriero and Masad Damha (McGill University) for their generous gift of synthetic branched RNA substrates.

Received August 1, 2016; accepted September 8, 2016.

\section{REFERENCES}

Acharya S, Földesi A, Chattopadhyaya J. 2003. The $\mathrm{p} K_{\mathrm{a}}$ of the internucleotide $2^{\prime}$-hydroxyl group in diribobucleoside $\left(3^{\prime}-5^{\prime}\right)$ monophosphates. J Org Chem 68: 1906-1910.

Arenas J, Hurwitz J. 1987. Purification of a RNA debranching activity from HeLa cells. J Biol Chem 262: 4274-4279.

Armakola M, Higgins MJ, Figley MD, Barmada SJ, Scarborough EA, Diaz Z, Fang X, Shorter J, Krogan NJ, Finkbeiner S, et al. 2012. 
Inhibition of RNA lariat debranching enzyme suppresses TDP-43 toxicity in ALS disease models. Nat Genet 44: 1302-1309.

Bitton DA, Rallis C, Jeffares DC, Smith GC, Chen YYC, Codlin S, Marguerat S, Bähler J. 2014. LaSSO, a strategy for genome-wide mapping of intronic lariats and branch points using RNA-seq. Genome Res 24: 1169-1179.

Buratti E. 2015. Functional significance of TDP-43 mutations in disease. Adv Genetics 91: 1-53.

Carriero S, Damha MJ. 2002. Solid-phase synthesis of branched oligonucleotides. Curr Protoc Nucleic Acid Chem 4: 14.

Chapman KB, Boeke JD. 1991. Isolation and characterization of the gene encoding yeast debranching enzyme. Cell 65: 483-492.

Garrey SM, Katolik A, Prekeris M, Li X, York K, Bernards S, Fields S, Zhao R, Damha MJ, Hesselberth JR. 2014. A homolog of lariatdebranching enzyme modulates turnover of branched RNA. RNA 20: $1337-1348$.

Herrick D, Parker R, Jacobson A. 1990. Identification and comparison of stable and unstable mRNAs in Saccharomyces cerevisiae. Mol Cell Biol 10: 2269-2284.

Hopfner KP, Karcher A, Craig L, Woo TT, Carney JP, Tainer JA. 2001. Structural biochemistry and interaction architecture of the DNA double-strand break repair Mre11 nuclease and Rad50-ATPase. Cell 105: 473-485.

Katolik A, Johnsson R, Montemayor E, Lackey JG, Hart PJ, Damha MJ. 2014. Regiospecific solid-phase synthesis of branched oligoribonucleotides that mimic intronic lariat RNA intermediates. $\mathrm{J} \mathrm{Org}$ Chem 79: 963-975.

Keppetipola N, Shuman S. 2006. Distinct enzymic functional groups are required for the phosphomonoesterase and phosphodiesterase activities of Clostridium thermocellum polynucleotide kinase/phosphatase. J Biol Chem 281: 19251-19259.

Keppetipola N, Shuman S. 2007. Characterization of the $2^{\prime}, 3^{\prime}$-cyclic phosphodiesterase activities of Clostridium thermocellum polynucleotide kinase-phosphatase and bacteriophage $\lambda$ phosphatase. Nucleic Acids Res 35: 7721-7732.

Keppetipola N, Shuman S. 2008. A phosphate-binding histidine of binuclear metallophosphodiesterase enzymes is a determinant of $2^{\prime}, 3^{\prime}$ cyclic nucleotide phosphodiesterase activity. J Biol Chem 283: 30942-30949.

Khalid F, Damha MJ, Shuman S, Schwer B. 2005. Structure-function analysis of yeast RNA debranching enzyme (Dbr1), a manganese-dependent phosphodiesterase. Nucleic Acids Res 33: 6349-6360.

Matange N, Podobnik M, Visweswariah SS. 2015. Metallophosphoesterases: structural fidelity with functional promiscuity. Biochem J 467: 201-216.

Montemayor EJ, Katolik A, Clark NE, Taylor AB, Scheurmann JP, Combs DJ, Johnsson R, Holloway SP, Stevens SW, Damha MJ, et al. 2014. Structural basis of lariat RNA recognition by the intron debranching enzyme Dbr1. Nucleic Acid Res 42: 10845-10855.
Nam K, Hudson RH, Chapman KB, Ganeshan K, Damha MJ, Boeke JD. 1994. Yeast lariat debranching enzyme. Substrate and sequence specificity. J Biol Chem 269: 20613-20621.

Nam K, Lee G, Trambley J, Devine SE, Boeke JD. 1997. Severe growth defect in a Schizosaccharomyces pombe mutant defective in intron lariat degradation. Mol Cell Biol 17: 809-818.

Padgett RA, Konarska MM, Grabowski PJ, Hardy SF, Sharp PA. 1984. Lariat RNAs as intermediates and products in the splicing of messenger RNA precursors. Science 225: 898-903.

Podobnik M, Tyagi R, Matange N, Dermol U, Gupta AK, Mattoo R, Seshadri K, Visweswariah SS. 2009. A mycobacterial cyclic AMP phosphodiesterase that moonlights as a modifier of cell wall permeability. J Biol Chem 284: 32846-32857.

Qin D, Huang L, Wlodaver A, Andrade J, Staley JP. 2016. Sequencing of lariat termini in S. cerevisiae reveals $5^{\prime}$ splice sites, branch points, and novel splicing events. RNA 22: 237-253.

Ruskin B, Green MR. 1985. An RNA processing activity that debranches RNA lariats. Science 229: 135-140.

Ruskin B, Krainer AR, Maniatis T, Green MR. 1984. Excision of an intact intron as a novel lariat structure during pre-mRNA splicing in vitro. Cell 38: 317-331.

Shenoy AR, Capuder M, Draskovic P, Lamba D, Visweswariah SS, Podobnik M. 2007. Structural and biochemical analysis of the Rv0805 cyclic nucleotide phosphodiesterase from Mycobacterium tuberculosis. J Mol Biol 365: 211-225.

Stepankiw N, Raghavan M, Fogarty EA, Grimson A, Pleiss JA. 2015. Widespread alternative and aberrant splicing revealed by lariat sequencing. Nucleic Acids Res 43: 8488-8501.

Tago N, Katolik A, Clark NE, Montemayor EJ, Seio K, Sekine M, Hart PJ, Damha MJ. 2015. Design, synthesis, and properties of phosphoramidate $2^{\prime}, 5^{\prime}$-linked branched RNA: toward the rational design of inhibitors of the RNA lariat debranching enzyme. J Org Chem 80: 10108-10118.

Tanaka N, Schwer B. 2005. Characterization of the NTPase, RNA-binding, and RNA helicase activities of the DEAH-box splicing factor Prp22. Biochemistry 44: 9795-9803.

Velikyan I, Acharya S, Trifonova A, Földesi A, Chattopadhyaya J. 2001. The $\mathrm{p} K_{\mathrm{a}} \mathrm{s}$ of $2^{\prime}$-hydroxyl group in nucleosides and nucleotides. J Am Chem Soc 123: 2893-2894.

Wang LK, Smith P, Shuman S. 2013. Structure and mechanism of the $2^{\prime}, 3^{\prime}$ phosphatase component of the bacterial Pnkp-Hen1 RNA repair system. Nucleic Acids Res 41: 5864-5873.

Wong I, Lohman TM. 1993. A double-filter method for nitrocellulosefilter binding: application to protein-nucleic acid interactions. Proc Natl Acad Sci 90: 5428-5432.

Zheng S, Vuong BQ, Vaidyanathan B, Lin JY, Huang FT, Chaudhuri J. 2015. Non-coding RNA generated following lariat debranching mediates targeting of AID to DNA. Cell 161: 762-773. 

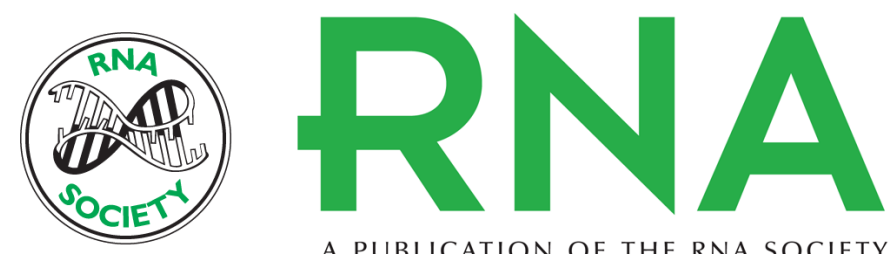

A PUBLICATION OF THE RNA SOCIETY

\title{
Mechanistic insights into the manganese-dependent phosphodiesterase activity of yeast Dbr1 with bis- $p$ -nitrophenylphosphate and branched RNA substrates
}

\author{
Beate Schwer, Fahad Khalid and Stewart Shuman
}

RNA 2016 22: 1819-1827 originally published online October 7, 2016

Access the most recent version at doi:10.1261/rna.058552.116

References This article cites 32 articles, 14 of which can be accessed free at:

http://rnajournal.cshlp.org/content/22/12/1819.full.html\#ref-list-1

Creative This article is distributed exclusively by the RNA Society for the first 12 months after the

Commons full-issue publication date (see http://rnajournal.cshlp.org/site/misc/terms.xhtml). After 12

License months, it is available under a Creative Commons License (Attribution-NonCommercial

4.0 International), as described at http://creativecommons.org/licenses/by-nc/4.0/.

Email Alerting Receive free email alerts when new articles cite this article - sign up in the box at the Service top right corner of the article or click here.

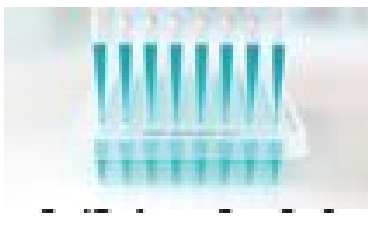

Providing Precise Solutions for your research.

To subscribe to $R N A$ go to:

http://rnajournal.cshlp.org/subscriptions 